\section{Wrong intraocular lens implant; learning from reported patient safety incidents}

SP Kelly and A Jalil

Eye (2011) 25, 730-734; doi:10.1038/eye.2011.22; published online 25 February 2011

Keywords: cataract; error; patient safety incident; biometry; wrong; reporting systems

Introduction

Cataract removal with intraocular lens (IOL) implantation is the most frequently undertaken elective surgical procedure performed in the United Kingdom. ${ }^{1}$ Although infrequent, one of the most prevalent confusions and potentially preventable errors is insertion of an incorrect or wrong IOL implant. ${ }^{2}$ IOL implantation errors usually result in a 'refractive surprise' wherein an unexpected/unintended post-operative refractive outcome occurs. A review of claims at the NHS Litigation Authority (NHSLA) found cataract care to be the largest share of litigation in ophthalmology and awarded the highest total damages. ${ }^{3}$ Claims relating to biometry errors / wrong IOL power were frequent in the NHSLA review. ${ }^{4}$ The litigation claims in cataract care in the United States are for similar reasons. ${ }^{5}$

Following several influential reports on patient safety, learning from clinical failure or incident is now widely regarded as a core principle underpinning improvement in patient safety. ${ }^{6}$ The National Patient Safety Agency (NPSA) (www.npsa.nhs.uk) was thus initiated. A patient safety incident (PSI) may be considered as an unintended or unexpected incident, which could have or did lead to harm for one or more patients. This is also referred to as an adverse event/incident or clinical error and includes 'near misses'. The NPSA includes a system for logging and gathering PSIs at national level following local reporting. NHS organizations in England and Wales now report to a National Reporting and Learning 
System (NRLS) repository database at the NPSA. Direct voluntary reporting to the NRLS is also possible. ${ }^{7}$

We reviewed PSI reports on the NRLS database at the NPSA to identify reported incidents in cataract surgery in England and Wales, where incorrect or wrong IOL implants were inserted or 'near misses' occurred. We aspired to identify any themes in these reports and to propose, if possible, measures to improve care.

\section{Methods}

NHS providers in England and Wales report patient safety incidents to the NPSA using local electronic submission processes. The information reported includes; clinical specialty, staff involved, location and time of the incident, degree of patient harm and free text description of the incident. All submitted PSI reports are made anonymous within the NRLS database. Access to review a NRLS search was granted to one of the authors (SPK). The intention was to identify incidents related to wrong IOL implantation.

A search of the NRLS database by keyword was undertaken in February 2010. All IOL related incidents reported to the database from 2003 up to 31 January 2010 were included. First, the free text fields were searched for instances of any of the terms; 'cataract', 'dioptre'; 'intraocular lens', 'IOL', plus any of the following terms present in the same PSI report: 'wrong', 'incorrect', 'error'.

All incident reports so retrieved from the NRLS database were reviewed to identify those related to wrong IOL implantation. Thematic analysis of such cases as reported was undertaken. Our analysis was based on the narrative details provided in such reports only.

\section{Results}

The NRLS database held 4256014 incident reports on the 31 January 2010 and of which 23871 were reported as related to the specialty of ophthalmology. A total of 22569 such ophthalmic PSI reports were from England, 1289 were from Wales and in 13 reports, the location of the incident was not identified. Of these PSI reports, 164 cases were identified where a wrong IOL incident was reported. The study period was from the initial roll out of the NRLS throughout 2003 up until the end of January 2010. Thematic analysis of the 164 incidents where incorrect IOL implantation occurred was undertaken with a view to identify sources of potential error. Table 1 provides the principle reasons by theme for the PSI reports. Multiple causal factors were not reported or specifically identifiable on any incident reports. There were no 'near miss' reports found. Broadly speaking, where a theme was discernable, safety
Table 1 Reasons for 'wrong' IOL implantation as reported

\begin{tabular}{rlr}
\hline & Thematic reasons for 'wrong' IOL implantation & $\begin{array}{c}\text { Number of } \\
\text { reports }\end{array}$ \\
\hline 1 & Inaccurate biometry & 29 \\
2 & Wrong IOL selection & 21 \\
3 & Transcription errors & 10 \\
4 & Handwriting misinterpretations & 7 \\
5 & Change in list order & 8 \\
6 & Right/left eye confusion & 5 \\
7 & Patient identification issues & 4 \\
8 & Misfiled biometry & 4 \\
9 & Wrong IOL written on theatre white board & 4 \\
10 & Optimal IOL power unavailable in stock & 3 \\
11 & Wrong IOL power implantation after & 3 \\
& complicated surgery & \\
12 & Wrong patient notes & 2 \\
13 & Communication errors & 2 \\
14 & No causal reasons documented & 62 \\
& Total patient safety incident reports & 164 \\
\hline
\end{tabular}

Table 2 Authors suggestions for reducing 'wrong' IOL implantation

- Follow best practice in capturing biometry and in IOL power calculations.

- Only rely on biometry source documents.

- Consider use of electronic patient records.

- Reduce potential for handwriting misinterpretations of IOL powers. Write IOL power required clearly and in full. Consider circling or highlighting the correct IOL power required on the source IOL calculation print out page.

- Beware that abbreviation ' $\mathrm{D}$ ' for dioptre and ' - ' for minus may confuse.

- Avoid use of operating theatre 'white boards' for IOL powers selection.

- Use of cataract pre-operative checklist and 'time out'.

- Ensure adequate stock of IOLs ranges is in place in operating department.

issues include: problems obtaining accurate biometry; problems matching biometry to patients; problems matching correct IOL implant to correct patient and to laterality. In our opinion, several of the matters reported in the PSIs revealed themes, which may have preventable measures (Table 2). Errors as a result of relying on operating theatre white boards / swab board are such an example. Patients' details with the IOL power were written on the white board. However, either because of a change in surgical list order or otherwise, such details on whiteboards were not updated after the previous patient's surgery, the wrong lens power was thus implanted. Several errors occurred as a result of change 
in the order of the planned surgical list and mixing up of sequential patients and IOL powers. Transcription confusions included mixing up handwritten IOL powers. Examples of such handwritten transcription dioptres power errors included; '11' and '17' mixed up, '14' and '19' mixed up, '10.5' and '19.5' mixed up, '20' and '2D' mixed up, and ' +20 ' read as ' 12.0 ' (by three operating theatre staff). Several cases were because of biometry errors including use of incorrect biometry detail and including use of incorrect biometry formulae. Misfiling of biometry results in incorrect patient clinical records contributed to some such errors. Mixing of IOL powers for right and left eyes of patients and misidentification of correct patient at biometry visit examination also occurred. A biometry error attributed to failure to remove rigid contact lenses in adequate time before outpatient biometric examination was reported in one case. In several PSI reports, the wrong IOL had to be implanted because of depletion of IOL bank stock, with the correct IOL power not being available in stock on the day of elective surgery.

Other incidents occurred when cataract surgery was complicated by posterior capsular rupture and the IOL implant, which was implanted was of incorrect power.

Many incidents $(n=62)$ simply reported 'wrong IOL implantation' without further causation as to why the clinical error occurred. Such tantalisingly brief PSI reports without any further detail of where the breakdown of correct care occurred add little to the essence of patient safety incident reporting, the spirit of which is to consider causation wherever possible. Such brief reports mean no memory of what had gone wrong was retained, so any opportunity of learning was lost.

There were 47 reports where further surgical intervention was required. Of these, 46 patients underwent IOL exchange and one patient underwent supplementary secondary piggyback IOL insertion. In total, 16 out of 46 patients had IOL exchange surgery undertaken on the day of initial cataract surgery as the error was promptly picked up. A total of 30 patients had delayed or staged IOL exchange. In 16 cases, it was stated that no further action was needed as refractive outcome was felt to be within acceptable limits. The remaining 101 cases/reports did not provide details of refractive outcome after wrong IOL implantation and whether any further intervention was needed or not. NHS cataract surgery is almost always undertaken as an elective procedure and within the working week. No time factors were discernable from PSI reports except for reports of depletion of weekday IOL stock levels, which was because of the undertaking of additional weekend 'extra capacity activity'. No type of staff (ie rotating or locum or permanent staff) present factors were discernable from the PSI reports. Unexpected refractive results in patients who had previously undergone corneal refractive surgery were not reported.

\section{Discussion}

The NRLS database contained close to 24000 PSI reports related to ophthalmology by end of January 2010. Surgical confusions (ie wrong patient, wrong site or side surgery, wrong procedure or implant) are an infrequent, but important and preventable cause of patient morbidity in general and relevant to ophthalmic surgeons. ${ }^{2-5,8,9}$ The Department of Health in England consultation exercise on 'never events' states that insertion of an incorrect implant is an event that should never happen. ${ }^{10}$ Ophthalmologists and orthopaedic surgeons at the Veterans' Health Administration experienced the highest rate of preventable adverse events resulting not only from wrong site procedures, but seemingly high rates of incorrect medical device implantation. ${ }^{11}$

Under-reporting is widespread and recent studies found that only a minority of NHS incidents are reported. ${ }^{12}$ Medical staff have barriers to incident reporting and reporting is often nurse lead. ${ }^{13-15}$ Thus, this review should not be regarded as an analysis of all wrong IOL implant incidents occurring in NHS care during the study period. A weakness of our retrospective review is that root causation of such IOL misadventures is problematic as PSI causation is not described by reporters in a standard format, or at all. Rather PSI reports are provided in free text by the reporter and frequently contain anecdote-based opinion. Furthermore, the severity of patient harm is self-declared by the PSI reporter. There were many cases where patients required additional surgical procedures in the form of IOL exchange, but the degree of harm from the original PSI was labelled as 'none'. A further potential weakness of our analysis is that device related incidents in NHS care (such as opaque IOLs) are reported to the Medical and Healthcare Regulatory Authority.

Broadly speaking, safety issues found include: problems obtaining accurate biometry; problems matching biometry to patients; problems matching correct IOL implant to correct patient and to laterality. Each of these is a microcosm of the challenges of surgical care such as correctly capturing and then matching correct data to correct patients. On the basis of these themes, certain potential safety improvement advice can be advocated to seek to reduce wrong IOL implantation.

First, on a technology level, ocular biometry should be carried out with the most up to date, accurate biometric equipment and by appropriately trained staff. ${ }^{16}$ Advanced technology biometric instruments with improved signal to noise ratio incorporating anterior 
chamber depth measurements including third- and fourth-generation biometric formulae are available and should be used. With the increasingly routine use of optical biometry - when clinically possible- and modern IOL calculation formulae and optimisation of 'A constants' busy NHS cataract facilities with a variety of surgeons and biometry technicians should be able to achieve a refractive outcome within $+/-1$ dioptre of the target refractive outcome in $85 \%$ of cases. ${ }^{17}$

Second, many errors in IOL implantation reported were not complex technological issues, but occurred because of misfiling or misreading of biometry printouts, transcription mix-ups, and communication breakdowns between the operating surgeon and nursing staff. We suggest the following measures to attempt to reduce these risks: compare the fellow eye biometry and refraction; rely only on clinical source documents, such as the biometry instrument paper print out or digital output; consider circling or highlighting the IOL power desired on the biometry paper printout and if handwritten clinical records are in use, consider writing out the IOL implant power required clearly and in full. Furthermore, we suggest avoiding use of the abbreviation ' $\mathrm{D}$ ' (for dioptre) following the IOL implant dioptre power required, especially where handwritten clinical records are in use. Also, avoid use of handwritten ' + ' or ' - ' symbols. If a minus powered IOL is needed - especially where handwritten clinical records are in use-we suggest writing out the word 'minus' in full. An NRLS review also found poor handwriting to be a risk in the prescription of insulin. ${ }^{18}$ Electronic patient records (EPRs) may reduce error from poor handwriting. Linking EPRs directly to the biometry instrument output may provide a potential additional safety feature. In the light of the present PSI reports, we also suggest that surgical teams should be careful about or avoid relying on theatre whiteboards or pre-printed operating lists for correct IOL implant selection.

Third, is important in our experience to avoid, minimise or cope with distractions during cataract surgery. The Patient Safety First campaign ${ }^{19}$ and similar initiatives recommends both a team brief before surgery and taking 'time out' before starting the surgical procedure. The Royal College of Ophthalmologists (the College) provides broad guidance on patient safety for ophthalmologists. ${ }^{20,21}$ Specific safety guidance for cataract care are provided within the Cataract Surgery Guidelines. $^{16}$

Parallels are often drawn between healthcare and aviation. ${ }^{22,23}$ It is recommended that clinical staff could adopt several safety traits of aviation; error assumption, procedure standardisation, and institutionalised safety. ${ }^{24,25}$ Checklist use similar to aviation pre-flight checklists has gained additional support with the
World Health Organization's initiative 'Safe Surgery Saves Lives' (www.who.int/patientsafety/safesurgery/ en/index.html). Both surgical checklist use and team training has provided evidence of benefit. ${ }^{26-29}$ The Royal College of Ophthalmologists and the NPSA have launched a bespoke checklist for cataract surgery and requires confirmation before surgery that the correct IOL implant has been determined and is available. ${ }^{30}$ The American Academy of Ophthalmology has provided a performance improvement educational resource on reducing wrong site/wrong IOL surgery. ${ }^{31}$

In conclusion, this retrospective review of patient safety incidents as reported to a national database demonstrates that learning from such a database has some merit in cataract surgery. Specifically, we found that technological, social/organizational and team training factors have some roles in wrong IOL event causation. Some potential solutions to reduce these events are recommended. It is not possible for us to estimate the reduction in failures that might occur as a consequence of our recommendations. We welcome further suggestions from cataract teams on how best to reduce wrong IOL implant events.

\section{Summary}

\section{What was known before}

- Although infrequent, biometry/IOL implant error occasionally occurs.

- Wrong IOLs are a potent area of complaint and litigation.

- NHS patient safety incident reports are being collected into a central database from across England and Wales.

\section{What this study adds}

- A national database of patient safety incident reports can provide learning to staff involved with cataract care.

- There is room for improvement in patient safety incident reporting in cataract care as root causation of error is often lacking in such reports.

- Suggestions to reduce wrong IOL implantation based on incident reports received from the service are provided.

\section{Conflict of interest}

The authors declare no conflict of interest.

\section{Acknowledgements}

We thank Michael Surkitt-Parr at the NPSA for undertaking database searches. We thank Fran Watts, Patient Safety Lead, for surgery of the NPSA and Richard Smith, recent Chairman of Professional Standards Committee of the Royal College of Ophthalmologists, for comments on early drafts of the manuscript.

Contributors: SPK and AJ are responsible for drafting this manuscript. SPK is the guarantor of this article. 
Ethical approval: A data-sharing undertaking between SPK and the National Patient Safety Agency was undertaken in line with data protection procedures at the NPSA. No identifiable patient data were viewed in data analysis.

Provenance and peer review: Not commissioned; externally peer reviewed.

\section{References}

1 The Information Centre, hospital episode statistics, hesonline. Main procedures and interventions: 2000-2009. http:/ / www.hesonline.nhs.uk/Ease/servlet/ ContentServer?siteID = 1937\&categoryID = 215 .

2 Simon JW, Ngo Y, Khan S, Strogatz D. Surgical confusions in ophthalmology. Arch Ophthalmol 2007; 125: 1515-1522.

3 Ali N. A decade of clinical negligence in ophthalmology. BMC Ophthalmol 2007; 7: 20. doi: 10.1186/1471-2415-7-20.

4 Ali N, Little BC. Causes of cataract surgery malpractice claims in England 1995-2008. Br J Ophthalmol 2010: 182774. (e-pub ahead of print 30 August 2010; doi:10.1136/ bjo.2010.182774).

5 Brick DC. Risk management lessons from a review of 168 cataract surgery claims. Surv Ophthalmol 1999; 43(4): 356-360.

6 World Health Organization. WHO draft guidelines on adverse event reporting and learning systems. World Health Organization Press: Geneva, Switzerland, 2005. http://www.who.int/patientsafety/events/05/ Reporting_Guidelines.pdf (accessed October 2010).

7 National Patient Safety Agency. Report a patient safety incident here. http://www.nrls.npsa.nhs.uk/report-apatient-safety-incident/ (accessed October 2010).

8 Traquair H. Removal of the wrong eye. Br J Ophthalmol 1947; 31: 8-12.

9 Fraser SG, Adams W. Wrong site surgery. Br J Ophthalmol 2006; 90: 814-816.

10 Department of Health, London 2010 Expanding the list of never events. Accessed at http://www.dh.gov.uk/en/ Publicationsandstatistics/Publications/PublicationsPolicy AndGuidance/DH_120247 (accessed October 2010).

11 Neily J, Mills PD, Eldridge N, Dunn EJ, Samples C, Turner JR et al. Incorrect surgical procedures within and outside of the operating room. Arch Surg 2009; 144(11): 1028-1034.

12 Dixon-Woods M. Why is patient safety so hard? A selective review of ethnographic studies. J Health Serv Res Policy 2010; 15: 11-16.

13 Vincent CN, Stanhope N, Crowley-Murphy M. Reasons for not reporting adverse incidents: an empirical study. J Eval Clin Pract 1999; 5: 13-21.

14 Lawton R, Parker D. Barriers to incident reporting in a healthcare system. Qual Saf Health Care 2002; 11: 15-18.

15 Evans SM, Berry JG, Smith BJ, Esterman A, Selim P, $\mathrm{O}$ 'Shaughnessy $\mathrm{J}$ et al. Attitudes and barriers to incident reporting: a collaborative hospital study. Qual Saf Health Care 2006; 15: 39-43.

16 The Royal College of Ophthalmologists. Cataract Surgery Guidelines 2010. The Royal College of Ophthalmologists: London, 2010. Available at http://www.rcophth.ac.uk/page.asp?section $=451 \&$ sectionTitle $=$ Clinical + Guidelines (accessed October 2010)

17 Gale RP, Saldana M, Johnston RL, Zuberbuhler B, McKibbon M. Benchmark standards for refractive outcomes after NHS cataract surgery. Eye 2009; 23: 149-152.

18 Lamont T, Cousins D, Hillson R, Bischler A, Terblanche M. Safer administration of insulin: summary of a safety report from the National Patient Safety Agency. BMJ 2010; 341: c5269; doi:101136/bmjc5269.

19 Patient Safety First Campaign. Quick Guide to Briefing. The Patient Safety First Campaign: London, 2010. Available at http://www.patientsafetyfirst.nhs.uk/ashx/Asset.ashx?path $=/$ Intervention-support/Quick\%20guide\%20to\%20briefngs \%20and\%20debriefings.pdf. (accessed October 2010).

20 The Royal College of Ophthalmologists. Patient Safety in Ophthalmology; Ophthalmic Services Guidance. The Royal College of Ophthalmologists: London, 2008. Available at http:/ / www.rcophth.ac.uk/documents.asp?section=39 \&sectionTitle=Publications\&let $=P$ (accessed October 2010).

21 Kelly SP. Guidance on patient safety in ophthalmology from the royal college of ophthalmologists. Eye 2009; 23: 2143-2151.

22 Rutherford W. Aviation safety: a model for health care? Qual Saf Health Care 2003; 12: 162-163.

23 Hudson P. Applying the lessons of high risk industries to health care. Qual Saf Health Care 2003; 12(1 Suppl): i7-i12S.

24 Leape LL. Error in medicine. JAMA 1994; 272: 1851-1857.

25 Singh N. On a wing and a prayer: surgeons learning from the aviation industry. I R Soc Med 2009; 102: 360.

26 World Health Organization. Checklists save lives. Bull World Health Organ 2008; 86(7): 501-502.

27 Haynes AB, Weiser TG, Berry WR, Lipsitz SR, Breizat AH, Dellinger EP et al. A surgical safety checklist to reduce morbidity and mortality in a global population. N Engl J Med 2009; 360: 491-499.

28 Paull DE, Mazzia LM, Wood SD, Theis MS, Robinson LD, Carney $\mathrm{B}$ et al. Briefing guide study: preoperative briefing and postoperative debriefing checklists in the Veterans Health Administration medical team training program. Am J Surg 2010; 200(5): 620-623.

29 de Vries EN, Prins HA, Crolla RM, den Outer AJ, van Andel $\mathrm{G}$, van Helden $\mathrm{SH}$ et al. Effect of a Comprehensive Surgical Safety System on Patient Outcomes. N Engl J Med 2010; 363(20): 1928-1937.

30 WHO Surgical Safety Checklist: for cataract surgery only. National Patient Safety Agency: London, 2010. Available at http:/ /www.nrls.npsa.nhs.uk/resources/clinical-specialty/ surgery/?entryid45=74132 (accessed October 2010).

31 American Academy of Ophthalmology. Wrong Site/ Wrong IOL Performance Improvement CME. Accessed at http://one.aao.org/ce/educationalcontent/performance improvementcme.aspx (accessed October 2010). 